\title{
Desempenho, rendimento de carcaça e cortes nobres de frangos de corte alimentados com rações suplementadas com Solanum glaucophyllum
}

\section{Performance, carcass and noble cuts yield of broilers fed diets supplemented with Solanum glaucophyllum}

\author{
Flávio Medeiros Vieites ${ }^{1 *}$; Rodrigo Pereira Nalon²; Andreza Luzia Santos ${ }^{3}$; \\ Patricia Azevedo Castelo Branco ${ }^{4}$; Christiane Silva Souza ${ }^{5}$; Ricardo Vianna Nunes ${ }^{6}$; \\ Arele Arlindo Calderano ${ }^{7}$; Nelson Vital Monteiro de Arruda ${ }^{8}$
}

\section{Resumo}

\begin{abstract}
Objetivou-se avaliar o desempenho de frangos de corte entre 8 e 42 dias de idade, alimentados com rações suplementadas com 1,25-dihidroxicolecalciferol. Foram utilizados 648 frangos de corte, machos da linhagem Cobb $^{\circledR}$, distribuídos em seis tratamentos $(0,0 ; 0,5 ; 1,0 ; 1,5 ; 2,0 ;$ e $2,5 \mu \mathrm{g}$ de vitamina $\mathrm{D}_{3} / \mathrm{kg}$ de ração) em delineamento constituído de blocos casualizados, com seis repetições de 18 aves cada. Aos 8 e 42 dias de idade cada unidade experimental e as rações foram pesadas para avaliação do desempenho (consumo de ração - CR, ganho de peso - GP e conversão alimentar - CA). Posteriormente, três aves/repetição foram retiradas e abatidas para mensuração do peso absoluto, rendimentos de carcaça e de cortes nobres. As médias de CR, GP e CA foram: 3,68 kg, 2,29 kg e 1,60, respectivamente. Os rendimentos de carcaça, peito, coxa, sobrecoxa e asas foram respectivamente: 70,17\%, 37,09\%, $14,08 \%, 18,31 \%$ e $10,44 \%$. A inclusão de até $2,5 \mu \mathrm{g}$ de vitamina $\mathrm{D}_{3}$ ativa $/ \mathrm{kg}$ na ração não influenciou $(\mathrm{p}>0,05)$ o desempenho e os rendimentos de carcaça e cortes nobres de frangos de corte.
\end{abstract}

Palavras-chave: 1,25-dihidroxicolecalciferol, avicultura industrial, rendimento de carne

\footnotetext{
Abstract

The objective was to evaluate the performance of broilers from 8 to 42 days of age, fed with diets supplemented with active vitamin $\mathrm{D}_{3}$. It was used 648 broilers, males, $\mathrm{Cobb}{ }^{\circledR}$, distributed among six treatments $\left(0.0 ; 0.5 ; 1.0 ; 1.5 ; 2.0\right.$ and $2.5 \mathrm{D}_{3} / \mathrm{kg}$ of ration) using a randomized block design, with six repetitions of 18 birds each. At 8 and 42 days of age each experimental unit and the rations were weighted in order to evaluated the performance (feed intake - FI, weight gain - WG and feed conversion - FC). Later, three broilers/repetition were removed and slaughtered in order to measure the absolutely weight, carcass and noble cuts yield. The average carcass, breast, thighs, drumsticks and wings were

${ }^{1}$ Prof. do Programa de Pós-Graduação em Ciência Animal, PGCA, Universidade Federal de Mato Grosso, UFMT, Cuiabá, MT. Bolsista do Conselho Nacional de Desenvolvimento Científico e Tecnológico, CNPq. E-mail: fmvieites@yahoo.com.br

${ }^{2}$ M.e em Ciência Animal, UFMT, Cuiabá, MT. E-mail: rodrigopoxo@hotmail.com

${ }^{3}$ M.e em Zootecnia, Universidade Federal de Viçosa, UFV, Viçosa, MG. E-mail: 1sandreza@yahoo.com.br

${ }^{4}$ Prof ${ }^{a}$ da UFMT, Sinop, MT. E-mail: castelobranco78@hotmail.com

${ }^{5}$ M.e em Ciência Animal, UFMT, Cuiabá, MT. E-mail: christiane_s_souza@hotmail.com

${ }^{6}$ Prof. do Programa de Pós-Graduação em Zootecnia, Universidade Estadual do Oeste do Paraná, UNIOESTE, Marechal Rondon, PR. E-mail: nunesrv@hotmail.com

${ }^{7}$ Gerente de Produtos, Technofeed Ltda, Vinhedo, SP. E-mail: arele.calderano@technofeed.com.br

${ }^{8}$ Prof. da UFMT, Rondonópolis, MT. E-mail: nelsonarruda.vet@bol.com.br

* Autor para correspondência
} 
$70.17 \%, 37.09 \%, 14.08 \%, 18.31 \%$ and $10.44 \%$, respectively. The levels of active vitamin $\mathrm{D}_{3}$ did not influence ( $>0.05)$ performance, nor the carcass and noble cuts of broilers. Including up to $2.5 \mu \mathrm{g}$ active vitamin $\mathrm{D}_{3} / \mathrm{kg}$ in ration did not influence $(\mathrm{p}>0.05)$ the performance and carcass and noble cuts yield of broilers.

Key words: 1,25-dihydroxycholecalciferol, carcass yield, industry poultry

\section{Introdução}

A produção de frangos está presente em todo Brasil, impactando positivamente a economia dos estados nos quais a atividade vem se desenvolvendo. A alimentação das aves é o fator que mais onera o setor produtivo, representando aproximadamente $70,0 \%$ dos custos na avicultura industrial. Por essa razão, verifica-se uma tendência de expansão da atividade para o Centro-Oeste do país, região produtora de grãos, com o objetivo de reduzir os custos com a alimentação e possibilitar maior competitividade no mercado internacional.

O setor avícola brasileiro é caracterizado pela elevada eficiência produtiva, ocupando posição de destaque no cenário mundial. A alta produtividade se deve ao advento tecnológico, programas de melhoramento genético, e de manejo nutricional diferenciado. Neste contexto, programas alimentares específicos têm sido adotados, visando o suprimento adequado de nutrientes aos animais por meio de rações balanceadas. Dentre tais nutrientes, têm-se as vitaminas que são essenciais para o desenvolvimento animal, participando como cofatores em reações metabólicas e permitindo maior eficiência dos sistemas de síntese no organismo animal. A adição de complexos vitamínicos nas dietas de frangos de corte auxilia no seu desenvolvimento e melhora a eficiência da resposta imune. Félix, Maiorka e Sorbara (2009) salientaram que a maioria das vitaminas não são sintetizadas em quantidades suficientes para atender a velocidade da demanda fisiológica do organismo, sendo obtidas dos alimentos.

Dentre as vitaminas mais importantes no desenvolvimento das aves está a vitamina $\mathrm{D}$, que tem expressiva participação no metabolismo ósseo, sendo diretamente responsável pelo crescimento esquelético, que dá suporte às aves para obtenção do máximo desempenho produtivo (BRITO et al., 2010). Existem basicamente dois tipos de vitamina $D$, o ergocalciferol vegetal irradiado $\left(D_{2}\right)$ e o colecalciferol animal $\left(\mathrm{D}_{3}\right)$. Para suínos, as formas dietéticas de $\mathrm{D}_{2}$ e $\mathrm{D}_{3}$ são eficientemente utilizadas, contudo, as aves aproveitam 10 vezes mais a $D_{3}$ (BERTECHINI, 2006).

Nos últimos anos, a vitamina $\mathrm{D}_{3}$ e seus metabólitos (25-hidroxi-colecalciferol $\left(25(\mathrm{OH})_{2} \mathrm{D}_{3}\right)$ e 1,25-dihidroxicolecalciferol $\left(1,25(\mathrm{OH})_{2} \mathrm{D}_{3}\right)$ têm sido estudados na avicultura, com o propósito de investigar a interação entre nutrição, desempenho e características ósseas. Gonzales e Mendonça Júnior (2006) relataram que para as linhagens modernas de frangos de corte, e principalmente nos machos, a taxa de crescimento do tecido muscular é extremamente elevada, iniciando-se em uma fase muito precoce pós-eclosão sobre um suporte esquelético muito imaturo, podendo causar desordens locomotoras, comprometendo consequentemente o bem-estar e desempenho das aves.

Entretanto, existem na literatura poucos estudos específicos que sejam conclusivos relacionando a suplementação da vitamina $\mathrm{D}_{3}$ sobre o desempenho e as características da carcaça em frangos de corte, o que dificulta colocar em prática a concepção da recomendação ideal em dietas comerciais.

O objetivo com este trabalho foi avaliar o desempenho, o rendimento de carcaça e de cortes nobres de frangos de corte entre oito e 42 dias de idade, suplementados com níveis de vitamina $\mathrm{D}_{3}$ ativa oriunda da planta Solanum glaucophyllum. 


\section{Material e Métodos}

O experimento foi conduzido no setor de avicultura do Instituto Federal de Educação, Ciência e Tecnologia do Estado de Mato Grosso (IFMT), Campus São Vicente (CSV), no município de Santo Antônio de Leverger-MT, de setembro a outubro de 2011.

Foram utilizados 648 frangos de corte machos da marca comercial Cobb $\AA$. O delineamento adotado foi o de blocos casualizados com seis tratamentos: 0, 50, 100, 150, 200 e 250 gramas de Panbonis ${ }^{\circledR} /$ tonelada de ração, correspondendo a 0,$0 ; 0,5 ; 1,0 ; 1,5 ; 2,0$ e $2,5 \mu \mathrm{g}$ de vitamina $\mathrm{D}_{3}$ ativa/ $\mathrm{kg}$ de ração, respectivamente, com seis repetições. A unidade experimental (boxe) foi constituída por 18 aves. A distribuição em blocos ocorreu para que houvesse maior uniformidade nas parcelas do galpão experimental quanto ao posicionamento dos boxes. $\mathrm{O}$ produto comercial utilizado como fonte da vitamina $\mathrm{D}_{3}$ ativa (Panbonis ${ }^{\circledR}$, Technofeed, Suíça) foi incluído nas rações experimentais em substituição ao material inerte (areia), sendo composto de $10 \mathrm{mg} / \mathrm{kg}$ desta vitamina (Solanum glaucophyllum industrializada).

No período que antecedeu o experimento, as aves receberam vacinação contra a doença de Marek no incubatório e contra a doença de Newcastle no quinto dia de vida. No período de um a sete dias de idade, foram alimentadas com ração à vontade formulada para atender as exigências desta fase de criação. A ração e a água foram fornecidas utilizando comedouros tubulares infantis e bebedouros do tipo copo de pressão.

Aos oito dias de idade, foram pesadas e distribuídas uniformemente nos boxes. $\mathrm{O}$ peso médio dos pintinhos foi de $0,157 \mathrm{~kg}$. As aves foram alojadas em galpão de alvenaria, com mureta lateral, telado, pé direito de 3,0 metros, coberto com telhas de barro, cortinas laterais em toda a sua extensão e dois ventiladores suspensos e posicionados em mesma direção. Internamente o galpão foi subdividido em 36 boxes de 1,0 x 1,86 m (1,86 $\mathrm{m}^{2}$ ). A casca de arroz foi utilizada como material da cama aviária e colocado em cada boxe numa altura média de $10 \mathrm{~cm}$.

As aves foram submetidas ao regime de 24 horas de luz (natural + artificial) e as médias das temperaturas máxima e mínima e umidade relativa registradas durante todo o período experimental foram de 32,9 e $22,1^{\circ} \mathrm{C}$ e $42,3 \%$, respectivamente.

As dietas experimentais foram formuladas seguindo as recomendações de Rostagno et al. (2005), para as fases de crescimento e terminação (Tabela 1), sendo elaboradas substituindo o material inerte (areia) pelo produto comercial (vitamina $\mathrm{D}_{3}$ ativa) em diferentes níveis.

A ração foi pesada e armazenada em baldes escuros com tampa para posterior distribuição nos comedouros. Para avaliação do ganho de peso e conversão alimentar as aves foram pesadas no inicio e no término do período experimental, após serem submetidas a um jejum alimentar de duas horas no inicio e de quatro horas no término do período experimental. O consumo de ração por ave foi calculado da seguinte forma: (ração fornecida - sobra)/número de animais presentes no boxe, corrigido pela mortalidade quando necessário.

Após determinação do desempenho, três aves de cada repetição, com o peso médio da unidade experimental foram pesadas e encaminhadas ao frigorífico do IFMT/CSV, onde foram abatidas de acordo com os princípios éticos de experimentação animal. Após a insensibilização por eletrocussão, as aves foram submetidas a uma sangria pela veia jugular, e em seguida, depenadas, evisceradas, e resfriadas por um período de 12 horas. Posteriormente, retiraram-se os pés e as cabeças das carcaças, para pesagem das mesmas e realização dos cortes nobres: peito, coxa, sobrecoxa e asa. Foram avaliados o peso absoluto (g) e o rendimento (\%) das carcaças evisceradas e dos cortes nobres (peito, filé de peito, coxa + sobre coxa). Na determinação do rendimento, foi considerado o peso da carcaça limpa e eviscerada, 
em relação ao peso vivo do frango em jejum. Para foram feitos em relação ao peso da carcaça os cortes nobres, os cálculos dos rendimentos eviscerada.

Tabela 1. Rações experimentais e composição calculada das fases de 8 a 21, 22 a 35 e 36 a 42 dias de idade.

\begin{tabular}{|c|c|c|c|}
\hline Ingredientes (g/kg) & 8 a 21 & 22 a 35 & 36 a 42 \\
\hline Milho & 579,2 & 608,3 & 640,00 \\
\hline Farelo de soja & 350,6 & 314,8 & 279,00 \\
\hline Óleo de soja & 28,6 & 37,70 & - \\
\hline Gordura suína & - & - & 43,80 \\
\hline Fosfato bicálcico & 18,1 & 1,67 & 15,20 \\
\hline Calcário & 8,30 & 7,90 & 7,50 \\
\hline Sal Comum & 4,40 & 4,20 & 4,00 \\
\hline DL - Metionina (99\%) & 2,70 & 2,50 & 2,40 \\
\hline $\mathrm{L}-$ Lisina $\mathrm{HCl}(50,7 \%)$ & 3,40 & 3,30 & 4,00 \\
\hline L - Treonina (99\%) & 0,70 & 0,60 & 0,80 \\
\hline Cloreto de colina (60\%) & 1,00 & 1,00 & 1,00 \\
\hline Mistura Vitamínica e Mineral ${ }^{1,2}$ & 2,00 & 2,00 & 2,00 \\
\hline Virginiamicina $(10 \%)$ & 0,20 & 0,20 & - \\
\hline Salinomicina $(12 \%)$ & 0,50 & 0,50 & - \\
\hline Antioxidante $^{3}$ & 0,10 & 0,10 & 0,10 \\
\hline Areia lavada (inerte) & 0,20 & 0,20 & 0,20 \\
\hline \multicolumn{4}{|l|}{ Composição Calculada (\%) } \\
\hline Energia Metabolizável (Kcal) & 3050 & 3147 & 3200 \\
\hline Proteína bruta & 21,14 & 19,73 & 18,42 \\
\hline Cálcio & 0,90 & 0,84 & 0,78 \\
\hline Fósforo disponível & 0,45 & 0,42 & 0,39 \\
\hline Sódio & 0,22 & 0,21 & 0,20 \\
\hline Cloro & 0,30 & 0,29 & 0,27 \\
\hline Metionina + cistina digestível & 0,84 & 0,79 & 0,75 \\
\hline Lisina digestível & 1,19 & 1,10 & 1,05 \\
\hline Treonina digestível & 0,77 & 0,71 & 0,68 \\
\hline Triptofano digestível & 0,24 & 0,22 & 0,20 \\
\hline
\end{tabular}

${ }^{1}$ Suplemento Vitamina A - 5.546.000,0 UI/kg; Ferro - 24.800,00 mg; Selênio -150,00 mg; Vitamina D 3 - 1.339.000,00 UI/kg; Vitamina $\mathrm{K}_{3}-944,00 \mathrm{mg}$; Vitamina $\mathrm{B}_{1}-1.005,00 \mathrm{mg}$; Vitamina $\mathrm{B}_{6}-1245,00 \mathrm{mg}$; Ácido Pantotênico - 5.890,00 mg; Ácido Fólico - 495,00 mg; Cobre - 4.280,00 mg; Iodo - 500,00 mg; Vitamina $\mathrm{B}_{2}-2.250,00 \mathrm{mg}$; Vitamina $\mathrm{B}_{12}-6.000,00 \mathrm{mcg}$; Niacina - 15000,00 mg; B.H.T - 1.000,00 mg; Biotina - 50,00 mg; Manganês - 33.300,00 mg; Zinco - 25.680,00 mg; Vitamina E 12.430,00 UI/kg. ${ }^{2}$ Suplemento: Vitamina A - 2726,00UI/kg; Ferro - 24.800,00 mg; Selênio - 150,00 mg; Vitamina $\mathrm{D}_{3}-658300,00$ $\mathrm{UI} / \mathrm{kg}$; Vitamina $\mathrm{K}_{3}-464,00 \mathrm{mg}$; Vitamina $\mathrm{B}_{1}-502,00 \mathrm{mg}$; Vitamina $\mathrm{B}_{6}-623 \mathrm{mg}$; Ácido Pantotênico - 2940,00 mg; Ácido Fólico - 153,00 mg; Cobre - 4280,00 mg; Iodo - 500,00 mg; Vitamina $\mathrm{B}_{2}-1.125,00 \mathrm{mg}$; Vitamina $\mathrm{B}_{12}-3000,00 \mathrm{mcg}$; Niacina - 7500,00 mg; B.H.T - 1000,00 mg; Biotina - 15,00 mg; Manganês - 33.300,00 mg; Zinco - 25.680,00 mg; Vitamina E - 4.990,00 UI/kg. ${ }^{3}$ Butil-hidróxi-tolueno (BHT).

Fonte: Elaboração dos autores.

Os dados obtidos foram submetidos à análise de variância e comparação entre médias pelo Teste de Dunnett, considerando o nível de 5,0\% de probabilidade. As análises foram realizadas utilizando o software Sistema para Análises Estatísticas e Genética - SAEG (Universidade Federal de Viçosa, 2007). 
O modelo estatístico adotado foi o seguinte:

$Y i j=\mu+B j+N i+E_{i j}$, em que:

Yij = valor observado na unidade experimental do j-ésimo bloco que recebeu o i-ésimo tratamento;

$\mu=$ média geral;

$\mathrm{Bj}=$ efeito do bloco $\mathrm{j} ; 1 ; 2 ; 3 ; 4 ; 5 ; 6$;

$\mathrm{Ni}=$ efeito da suplementação da vitamina $\mathrm{D}_{3}$ ativa i;

$\mathrm{i}=0,50,100,150,200,250 \mathrm{~g} / \mathrm{ton} ;$

Eij= erro aleatório associado a cada observação.

\section{Resultados e Discussão}

O desempenho dos frangos de oito a 42 dias de idade estão apresentados na Tabela 2. Não houve diferença significativa $(\mathrm{p}>0,05)$ entre os níveis de suplementação com vitamina $\mathrm{D}_{3}$ ativa sobre as características avaliadas. O baixo consumo de ração no intervalo estudado refletiu em um peso médio abaixo do esperado para a marca comercial $\mathrm{Cobb}^{\circledR}$. Possivelmente as altas temperaturas registradas no período experimental influenciaram o consumo, prejudicando o desenvolvimento das aves. A média da temperatura máxima foi de 32,9 ${ }^{\circ} \mathrm{C}$, bem superior à temperatura recomendada pelo manual COBB-VANTRESS ${ }^{\circledR}$ (2009) para a fase de 22 a 42 dias de idade que está entre 18,0 a $26,0^{\circ} \mathrm{C}$. Os frangos de corte são muito susceptíveis a altas temperaturas ambientais, pois o elevado custo energético do seu crescimento associa-se a um aumento na produção de calor metabólico (OLIVEIRA et al., 2010). A diminuição do consumo de ração e do crescimento observada em aves mantidas sob estresse por calor têm sido considerada como aparente tentativa de redução da produção de calor corporal, inclusive do calor gerado pelo processo de digestão (OLIVEIRA NETO et al., 2000).

Dionello et al. (2002) afirmaram que os frangos de corte adultos são sensíveis a altas temperaturas, sendo que o desenvolvimento máximo ocorre entre 18 e $20^{\circ} \mathrm{C}$. Segundo Bertechini et al. (1991), o aumento de temperatura de 17,1 para $27,0^{\circ} \mathrm{C}$ provoca, em frangos de corte no período de 29 a 42 dias de idade, redução linear no consumo de ração com reflexo de redução, também linear, no ganho de peso.

Os dados deste estudo estão de acordo com os encontrados por Brito et al. (2010), ao avaliar quatro níveis de suplementação de vitamina D (16; 30; 70 e $110 \mu \mathrm{g} / \mathrm{kg}$ de ração) oriundos de duas fontes $\left(\mathrm{D}_{3}\right.$ e $25-\mathrm{OHD}_{3}$ ) para frangos de corte de 22 a 38 dias de idade. Os resultados dos autores demonstraram não haver interação $(\mathrm{p}>0,05)$ entre os níveis e as fontes de vitamina $\mathrm{D}$ para o desempenho das aves, nem efeito $(\mathrm{p}>0,05)$ sobre o desempenho no período de 1 a 38 dias de idade, verificando que os resultados constantes na literatura não revelam grandes alterações no desempenho atribuídas à suplementação de vitamina $\mathrm{D}$ na fase final de criação.

Cheng et al. (2004), avaliaram três níveis de inclusão de Solanum glaucophyllum $(1,0 ; 2,5$ e $5,0 \mathrm{~g} / \mathrm{kg}$ de ração) em dietas de frangos de corte de sete a 28 dias de idade com redução dos níveis de cálcio e fósforo disponível para $0,56 \%$ e $0,28 \%$, respectivamente, obtendo aumento no ganho de peso das aves. Os autores ainda alegaram que houve maior eficiência na absorção e utilização do fósforo e que o nível de inclusão de 5,0 g da planta/kg de ração pode ser uma alternativa viável para redução dos custos com a suplementação de cálcio e fósforo nas rações das aves, além de minimizar o impacto ambiental proveniente da exploração avícola. 
Tabela 2. Desempenho de frangos de corte alimentados com vitamina $\mathrm{D}_{3}$ ativa.

\begin{tabular}{llllllllll}
\hline \multirow{2}{*}{ Parâmetro } & \multirow{0}{*}{} & \multicolumn{9}{c}{ Níveis (g/ton) } & \multirow{2}{*}{ Média } & \multirow{2}{*}{ p-value } & \multirow{2}{*}{ CV (\%) } \\
\cline { 3 - 7 } & $\mathbf{0}$ & $\mathbf{5 0}$ & $\mathbf{1 0 0}$ & $\mathbf{1 5 0}$ & $\mathbf{2 0 0}$ & $\mathbf{2 5 0}$ & & & \\
\hline PI $(\mathrm{kg})$ & 0,156 & 0,156 & 0,157 & 0,156 & 0,157 & 0,156 & 0,156 & $>0,05$ & 0,72 \\
CR $(\mathrm{kg})$ & 3,762 & 3,676 & 3,681 & 3,682 & 3,610 & 3,689 & 3,683 & $>0,05$ & 3,13 \\
GP $(\mathrm{kg})$ & 2,313 & 2,279 & 2,278 & 2,287 & 2,283 & 2,310 & 2,291 & $>0,05$ & 2,89 \\
CA & 1,62 & 1,61 & 1,61 & 1,61 & 1,58 & 1,59 & 1,60 & $>0,05$ & 2,89 \\
\hline
\end{tabular}

PI = Peso inicial; $\mathrm{CR}=$ Consumo de ração; $\mathrm{GP}=$ Ganho de peso; $\mathrm{CA}=$ conversão alimentar. $\mathrm{CV}=$ coeficiente de variação. As médias não diferem estatisticamente do tratamento testemunha pelo teste Dunnett ao nível de $5 \%$ de probabilidade $(\mathrm{p}>0,05)$.

Fonte: Elaboração dos autores.

Neste estudo, os níveis de cálcio e fósforo disponível foram mantidos seguindo as recomendações de Rostagno et al. (2005) e os níveis mais elevados de vitamina $\mathrm{D}_{3}$ ativa não afetou o desempenho dos frangos. Segundo Brito et al. (2010), os efeitos positivos sobre o desempenho encontrados na primeira fase (1 a 21 dias) com a suplementação de vitamina $\mathrm{D}$, independentemente da fonte, quando incorporados na fase de crescimento e associados à redução na suplementação vitamínica em 20,0\% não foram acumulativos. Ou seja, níveis altos de suplementação de vitamina D não foram eficientes em melhorar o desempenho, considerando o período de 1 a 38 dias de idade das aves. A fase inicial é mais susceptível a alterações mediante a suplementação de vitamina $\mathrm{D}$, pois é o período em que ocorre alta taxa de crescimento do tecido esquelético, além da imaturidade do trato digestório - digestão e absorção de lipídios e compostos lipossolúveis (GOFF, 2006).

Os resultados obtidos para desempenho estão de acordo com a maioria dos estudos que envolvem vitamina $\mathrm{D}$, ou seja, quando níveis de cálcio e/ou fósforo disponível estão dentro de limites de deficiência marginal ou atendendo às necessidades nutricionais, não são verificados efeitos diretos do incremento da suplementação de vitamina D (EDWARDS JÚNIOR, 2002; FRITTS; WALDROUP, 2005). Por outro lado, respostas mais sensíveis no desempenho foram observadas em condições de níveis reduzidos de cálcio e fósforo (RAO et al., 2006), podendo haver recuperação do desempenho através da otimização nos processos de absorção e metabolismo desses elementos (NAHM, 2007).

Goodgame et al. (2011) trabalharam com frangos de corte entre sete e 21 dias avaliando duas fontes de 25-hidroxicolecalciferol $(0 ; 2,5$; 5,$0 ; 10,0 ; 20,0 ; 40,0 ; 60,0$ e $80 \mu \mathrm{g} / \mathrm{kg}$ ) em rações a base de milho e farelo de soja deficientes em vitamina $\mathrm{D}_{3}$ e com níveis de $\mathrm{Ca}$ e $\mathrm{P}$ abaixo do recomendado. Os autores concluíram que o maior ganho de peso ocorrido nessa fase foi com o nível de $20,0 \mu \mathrm{g} / \mathrm{kg}$, independente da fonte de 25-hidroxicolecalciferol.

Khan et al. (2010) observaram que aos 42 dias de idade, frangos de corte alimentados com dietas suplementadas com $3.500 \mathrm{UI} / \mathrm{kg} \quad(87,5$ $\mu \mathrm{g} / \mathrm{kg}$ ) de vitamina $\mathrm{D}_{3}$ inativa tiveram ganho de peso significativamente maior $(p<0,05)$ que aqueles que receberam 200, 1500 e $2500 \mathrm{UI} / \mathrm{kg}$ da vitamina. Os autores ressaltaram que ao trabalhar com redução dos níveis de cálcio e fósforo o requerimento nutricional de vitamina $\mathrm{D}_{3}$ das aves aumenta consideravelmente.

Os níveis de vitamina $\mathrm{D}_{3}$ ativa não influenciaram $(p>0,05)$ os rendimentos de carcaça e cortes nobres das aves abatidas aos 42 dias de idade. As médias de rendimentos de carcaça, peito, coxa, sobrecoxa e asa foram: $70,17 \% ; 37,09 \% ; 14,08 \% ; 18,31 \%$ e $10,44 \%$, respectivamente, Tabela 3 . Esses resultados, estão 
de acordo com os obtidos por Brito et al. (2010) que avaliaram o rendimento de carcaça de frangos de corte nas fases de crescimento (22-38 dias) e de terminação (39-45 dias).

Tabela 3. Rendimento de carcaça e de cortes nobres de frangos de corte machos aos 42 dias de idade.

\begin{tabular}{|c|c|c|c|c|c|c|c|c|c|}
\hline \multirow{2}{*}{ Variável } & \multicolumn{6}{|c|}{ Suplementação de vitamina $D_{3}\left(g_{\text {ton }}{ }^{-1}\right)$} & \multirow{2}{*}{ Média } & \multirow{2}{*}{ p-value } & \multirow{2}{*}{$\begin{array}{l}\text { CV } \\
(\%)\end{array}$} \\
\hline & $\mathbf{0}^{1}$ & 50 & 100 & 150 & 200 & 250 & & & \\
\hline \multicolumn{10}{|c|}{ Peso médio aos 42 dias } \\
\hline Peso absoluto (kg) & 2,469 & 2,436 & 2,435 & 2,444 & 2,440 & 2,466 & 2,448 & $>0,05$ & 2,60 \\
\hline \multicolumn{10}{|c|}{ Carcaça } \\
\hline Peso absoluto (kg) & 1,722 & 1,711 & 1,708 & 1,722 & 1,712 & 1,735 & 1,718 & $>0,05$ & 2,65 \\
\hline Peso relativo $(\%)$ & 69,71 & 70,25 & 70,14 & 70,43 & 70,15 & 70,33 & 70,17 & $>0,05$ & 0,94 \\
\hline \multicolumn{10}{|c|}{ Peito } \\
\hline Peso absoluto (kg) & 0,638 & 0,633 & 0,639 & 0,636 & 0,632 & 0,647 & 0,637 & $>0,05$ & 3,25 \\
\hline Peso relativo $(\%)$ & 37,04 & 37,00 & 37,40 & 36,96 & 36,87 & 37,27 & 37,09 & $>0,05$ & 2,24 \\
\hline \multicolumn{10}{|c|}{ Coxa } \\
\hline Peso absoluto (kg) & 0,248 & 0,241 & 0,240 & 0,239 & 0,237 & 0,246 & 0,242 & $>0,05$ & 4,08 \\
\hline Peso relativo (\%) & 14,41 & 14,07 & 14,07 & 13,90 & 13,85 & 14,16 & 14,08 & $>0,05$ & 3,34 \\
\hline \multicolumn{10}{|c|}{ Sobrecoxa } \\
\hline Peso absoluto (kg) & 0,315 & 0,310 & 0,315 & 0,318 & 0,313 & 0,318 & 0,315 & $>0,05$ & 2,85 \\
\hline Peso relativo (\%) & 18,28 & 18,09 & 18,44 & 18,45 & 18,30 & 18,30 & 18,31 & $>0,05$ & 2,71 \\
\hline \multicolumn{10}{|c|}{ Asas } \\
\hline Peso absoluto (kg) & 0,180 & 0,179 & 0,178 & 0,179 & 0,179 & 0,182 & 0,180 & $>0,05$ & 3,12 \\
\hline Peso relativo (\%) & 10,45 & 10,47 & 10,40 & 10,42 & 10,44 & 10,49 & 10,44 & $>0,05$ & 2,40 \\
\hline
\end{tabular}

${ }^{1}$ Tratamento testemunha. $\mathrm{CV}=$ Coeficiente de variação. As médias não diferem estatisticamente do tratamento testemunha pelo teste Dunnett ao nível de $5 \%$ de probabilidade $(\mathrm{p}>0,05)$.

Fonte: Elaboração dos autores.

Entretanto, Korver (2005) observou rendimentos maiores de carcaça e de peito em frangos de corte aos 42 dias de idade mediante a suplementação de vitamina $\mathrm{D}_{3}$ juntamente com a $25(\mathrm{OH}) \mathrm{D}_{3}$, constatando ainda uma maior densidade cortical das tíbias com a redução de incidência de anomalias ósseas.

Os níveis de vitamina $\mathrm{D}_{3}$ ativa estudados $(0,0 ; 0,5$; 1,$0 ; 1,5 ; 2,0$ e 2,5 $\mu \mathrm{g} / \mathrm{kg}$ de ração) não influenciaram as características de carcaça avaliadas. É possível que tenha ocorrido uma melhora na mineralização óssea das aves. A suplementação de vitamina $\mathrm{D}_{3}$ nas rações de frangos de corte pode estar diretamente relacionada a uma diminuição de perdas de carcaças em frigoríficos em função de fraturas e/ou desordens locomotoras.
$\mathrm{Na}$ avicultura industrial, as condenações de carcaças e diminuições de rendimentos de cortes nobres decorrem principalmente pelas fraturas ocorridas durante as operações de apanha, transporte e abate. Tais fraturas afetam a operação dos equipamentos de evisceração automática e desossa, influenciando a velocidade na linha de processamento, e ocasionando perdas econômicas. Ferket et al. (2009) estudaram características ósseas e propriedades biomecânicas em perus de 0 a 20 semanas de idade. Os autores concluíram que a suplementação com minerais orgânicos (Zn, Mn e $\mathrm{Cu}$ ) em associação com 25-hidroxicolecalciferol (92 $\mu \mathrm{g} / \mathrm{kg}$ de ração) podem diminuir a incidência de desordens locomotoras associadas com o rápido crescimento, beneficiando a fase de crescimento e 
terminação das aves.

Desta forma, mais estudos são necessários para se conhecer as respostas das aves consumindo níveis crescentes de vitamina $\mathrm{D}_{3}$ ativa e em quais condições a sua suplementação poderia melhorar o rendimento de carcaça e de cortes nobres de frangos de corte.

\section{Conclusão}

A suplementação até $2,5 \mu \mathrm{g}$ de vitamina $\mathrm{D}_{3}$ ativa/kg de ração não influenciou o desempenho e os rendimentos de carcaça e de cortes nobres dos frangos de corte.

\section{Agradecimentos}

Ao Conselho Nacional de Desenvolvimento Científico e Tecnológico (CNPq) pela concessão do financiamento do projeto de pesquisa. Ao Instituto Federal de Educação, Ciência e Tecnologia do Estado de Mato Grosso (IFMT) - Campus São Vicente pela infraestrutura fornecida. A Evonik Brasil pela doação dos aminoácidos e a Technofeed Ltda. pela disponibilização do produto comercial Panbonis ${ }^{\circledR}$, utilizado no experimento.

\section{Referências}

BERTECHINI, A. G.; ROSTAGNO, H. S.; SILVA, M. A.; OLIVEIRA, A. L. G. Efeitos da temperatura ambiente e nível de energia da ração sobre o desempenho e a carcaça de frangos de corte. Revista Brasileira de Zootecnia, Viçosa, MG, v. 20, n. 3, p. 218-228, 1991.

BERTECHINI, A. G. Nutrição de monogástricos. Lavras, MG: UFLA, 2006. 301p.

BRITO, J. A. G.; BERTECHINI, A. G.; FASSANI, E. J.; RODRIGUES, P. B.; LIMA, E. M. C.; MENEGHETTI, C. Efeito da vitamina $\mathrm{D}_{3}$ e 25-hidroxi-colecalciferol sobre o desempenho, o rendimento de carcaça e a morfologia intestinal de frangos de corte. Revista Brasileira de Zootecnia, Viçosa, MG, v. 39, n. 12, p. 2656-2663, 2010. CHENG, Y. H.; GOFF, J. P.; SELL, J. L.; DALLORSO, M. E.; GIL, S.; PAWLAK, S. E.; HORST, R. L. Utilizing
Solanum glaucophyllum alone or phytase to improve phophorus utilization in broilers. Poultry Science, Champaign, v. 83, n. 3, p. 406-413, 2004.

COBB-VANTRESS Inc. Manual de manejo de frangos de corte. Arkansas: [s.n.], 2009. 70 p.

DIONELLO, N. J. L.; MACARI, M.; FERRO, J. A.; RUTZ, F.; FERRO, M. I. T.; FURLAN, L. R. Respostas fisiológicas associadas à termotolerância em pintos de corte de duas linhagens por exposição a altas temperaturas. Revista Brasileira de Zootecnia, Viçosa, MG, v. 31, n. 1, p. 79-85, 2002.

EDWARDS JÚNIOR, H. M. Studies on the efficiency of cholecalciferol and derivatives for stimulating phytate utilization in broilers. Poultry Science, Champaign, v. 81, n. 7, p. 1026-1031, 2002.

FÉLIX, A. P.; MAIORKA, A.; SORBARA, J. O. Níveis vitamínicos para frangos de corte. Ciência Rural, Santa Maria, v. 39, n. 2, p. 619-626, 2009.

FERKET, P. R.; OVIEDO-RONDÓN, E. O.; MENTE, P. L.; BOHÓRGUEZ, D. V.; SANTOS JUNIOR, A. A.; GRIMES, J. L.; RICHARDS, J. D.; DIBNER, J. J.; FELTS, V. Organic trace minerals and 25-hydroycholecalciferol affect performance characteristics, leg abnormalities, and biomechanical properties of leg bones of turkeys. Poultry Science, Champaign, v. 88, n. 1, p. 118-131, 2009.

FRITTS, C. A.; WALDROUP, P. W. Comparasion of cholecalciferol and 25-hydroxychloecalciferol in broilers diets designed to minimize phosphorus excretion. Journal Applied Poultry Research, Champaign, v. 14, n. 1, p. 156-166, 2005.

GOFF, J. P. Distúrbios do metabolismo dos carboidratos e da gordura. In: REECE, W. O. D. Fisiologia dos animais domésticos. 12. ed. Rio de Janeiro: Guanabara Koogan, 2006. $926 \mathrm{p}$.

GONZALES, E.; MENDONÇA JÚNIOR, C. X. Problemas locomotores em frangos de corte. In: SIMPÓSIO BRASIL SUL DE AVICULTURA, 7., 2006, Chapecó. Anais... Chapecó: Núcleo de Médicos Veterinários, 2006. p. 79-93.

GOODGAME, S. D.; MUSSINI, F. J.; LU, C.; BRADLEY, C. D.; WATKINS, S. E.; WALDROUP, P. W. Evaluation of a fermentation source of 25-hydroxy in broilers diets. International Journal of Poultry Science, Faisalabad, v. 10, n. 4, p. 295-299, 2011.

KHAN, S. H.; SHAHID, R.; MIAN, A. A.; SARDAR, R.; ANJUM, M. A. Effect of the level of cholecalciferol supplementation of broiler diets on the performance and tibial dyscondroplasia. Journal of Animal Physiology and Animal Nutrition, Chichester, v. 9, n. 5, p. 584-593, 2010. 
KORVER, D. Research, analytical techniques and practical experiences using $\mathrm{HyD}^{\mathrm{TM}}$. In: ARKANSAS NUTRITION CONFERENCE, 2005, Arkansas. Proceedings...Arkansas: [s.n.], 2005. 12 p.

NAHM, K. H. Efficient phosphorus utilization in poultry feeding to lessen the environmental impact of excreta. World's Poultry Science Journal, Cambridge, v. 63, n. 4, p. 625-654, 2007.

OLIVEIRA NETO, A. R.; OLIVEIRA, R. F. M.; DONZELE, J. L.; ROSTAGNO, H. S.; FERREIRA, R. A.; MAXIMIANO, H. C.; GASPARINO, E. Efeito da temperatura ambiente sobre o desempenho e características de carcaça de frangos de corte alimentados com dieta controlada e dois níveis de energia metabolizável. Revista Brasileira de Zootecnia, Viçosa, MG, v. 29, n. 1, p. 183-190, 2000.

OLIVEIRA, W. P.; OLIVEIRA, R. F. M.; DONZELE, J. L.; GOMES, P. C.; MARTINS, M. S.; ASSIS, A. P. Redução do nível de proteína bruta em rações para frangos de corte em ambiente de estresse por calor. Revista Brasileira de Zootecnia, Viçosa, MG, v. 39, n. 5, p. 1092-1098, 2010.

RAO, S. V. R.; RAJU, M. V. L. N.; PANDA, A. K.; SUNDER, G. S.; SHARMA, R. P. Effect of high concentrations of cholecalciferol on growth, bone mineralization and mineral retention in broiler chicks fed suboptimal concentrations of calcium and nonphytate phosphorus. Journal of Applied Poultry Research, Champaign, v. 15, n. 4, p. 493-501, 2006.

ROSTAGNO, H. S.; ALBINO, L. F. T.; DONZELE, J. L.; GOMES, P. C. OLIVEIRA, R. F.; LOPES, D. C.; FERREIRA, A. S.; BARRETO, S. L. T. Tabelas brasileiras para suínos e aves: composição de alimentos e exigências nutricionais. 2. ed. Viçosa, MG: Editora UFV, 2005. $186 \mathrm{p}$.

UNIVERSIDADE FEDERAL DE VIÇOSA - UFV. SAEG - Sistema de análise estatística e genética. Versão 9.1. Viçosa, MG: UFV, 2007. 
